UDC 615.072: 543.42.062

DOI: $10.15587 / 2519-4852.2020 .221721$

\title{
DEVELOPMENT OF AN ADVANCED STRATEGY ON THE ASSAY METHOD TRANSFER
}

\author{
N. Volovyk, D. Leontiev, V. Petrus, O. Gryzodub, Yu. Pidpruzhnykov
}

\begin{abstract}
Aim. The paper intends to frame and pilot the optimised science-based principles of the assay transfer. Materials and methods. The research was performed on desloratadine film-coated tablets, using an analytical balance Mettler Toledo XP 205DR and Class A volumetric glassware. Absorbance readings were measured on a $U V$-Vis spectrophotometer Lambda 25.

Results and discussion. The concept of method transfer that complements the conventional approach to validation with the lifecycle initiative and the metrological base of the State Pharmacopoeia of Ukraine was substantiated, following which the transfer of the spectrophotometric procedure for desloratadine assay was conducted. For the batch intended for the transfer, the budget of analytical and technological variability was balanced. The deviation of a single assay result from the grand mean was used as the criterion for accuracy in the transfer. The requirement for the one-sided confidence interval for assay result runs not to exceed the target uncertainty of the procedure was used as the criterion for precision. The control strategy requirements for variability sources and the analytical target profile requirements for precision and accuracy were met in the receiving unit.

Conclusion. The paper discusses the premise and advocates an alternative approach to the method transfer. Precision is proposed not to study during the transfer (in the short-term experiment) but assess from the stability data (in the long-term experiment). Compliance with the normal analytical practice (the maximum permissible variability attributed to analysts and analytical instruments) allows narrowing down the transfer design to the confirmation in the minimal experiment that the amplitude of variability sources lies within the predefined range Keywords: analytical procedure transfer, lifecycle, acceptance criteria, measurement uncertainty, desloratadine tablets
\end{abstract}

Copyright (C) 2020, N. Volovyk, D. Leontiev, V. Petrus, O. Gryzodub, Yu. Pidpruzhnykov.

This is an open access article under the CC BY license (http://creativecommons.org/licenses/by/4.0).

\section{Introduction}

Inextricably linked with validation, transfer constitutes an essential step in the lifecycle of analytical procedures, during which the intrinsic procedural knowledge gained in the development laboratory (sending unit, or SU) is passed to another laboratory (receiving unit, or RU) for subsequent routine use. Not until the transfer is successfully completed can the RU perform an analytical procedure.

The transfer of the analytical procedure is considered successful if the transferred knowledge is translated into an effective analytical control strategy, and the RU demonstrates an ability to run the procedure as intended against the predefined acceptance criteria continuously [1]. With that, it is necessary to keep in mind that processes change over time, and as with validation, the transfer projects a static rather than full picture. Therefore, even after successfully completing the transfer, the procedure performance should be monitored [2].

Acceptance criteria and performance characteristics of analytical procedures should be defined so that the scientifically substantiated and risk-based decisions about the ability of analytical procedures to consistently satisfy an analytical target profile (ATP), including requirements for the target measurement uncertainty, could be made [3]. Statistical methods for analysing collected data and recommendations for addressing deviations and handling non-compliant data should be provided.

The appropriate and efficient organisation of the transfer is crucial. However, official guidelines mainly contain general recommendations with a few examples of the experiment design and acceptance criteria that are either insufficiently justified or not suitable universally $[4,5]$.

What all procedures have in common is that they primarily tend to stick to either a classical or lifecycle approach. However, irrespective of which of them is taken, for the drug products to circulate globally, the procedure transfer should comply with the ICH recommendations.

The classical approach is built on the widely accepted ICH Q2(R1) guideline on validation of analytical procedures [6] whereby the transfer is considered an individual process though based on validation results. The validation concept described therein aims to show that the various performance characteristics (accuracy, linearity, precision, etc.) meet the predefined criteria with little consideration of how they relate to the overall uncertainty and whether they are acceptable or not [3]. As a consequence, the followers of the classical approach tend to run validation and transfer as a check-box exercise, without accounting for the sources of variability and further use of the analytical procedure, which often re- 
sults in failure for the RU to run the procedure as aimed and the need for repeated costly testing to identify the root cause and prevent recurrence [7].

The USP Expert Panel moved an alternative approach [3] and drafted the general chapter Analytical Procedure Life Cycle $<1220\rangle[8,9]$ that encompasses all the events designed to confirm the suitability of the analytical procedure for the intended use that occur throughout the entire life of the procedure, considering them under the framework of validation. It allows for a significant improvement to the conventional approach to validation. In the context of the procedure lifecycle, the transfer falls under Stage 2 Performance Qualification, which is closely connected with Stages 1 and 3 -Design and Continued Performance Verification, respectively. The approach championed by the USP concentrates on the understanding and control over variability sources at all stages of the procedure lifecycle. Obviously, the concept of the procedure lifecycle has distinct advantages. Yet, the performance characteristics recommended by ICH have not been translated into criteria although their importance has been stressed.

The State Pharmacopoeia of Ukraine (SPhU) developed the guidelines on validation of analytical procedures and statistical tools $[10,11]$ that rely on the classical approach but rest upon the concept of uncertainty [12], the application of which is driven by the standardisation rules adopted in the pharmaceutical sector. All ICH recommendations for performance characteristics were translated into the acceptance criteria based on the risk assessment of making wrong accept/reject decisions [13]. Consequently, it can be argued that the SPhU approach enables us to fulfil the primary task of validation, i.e. to demonstrate the fitness of the analytical procedure for the intended purpose. Hereinafter under the SPhU approach, we understand all recommendations of the $\mathrm{SPhU}$ that were published either in the compendia or journal Farmacom (the medium for the introduction and public discussion of the SPhU initiatives).

Introduced in 2004, the SPhU approach anticipated some aspects of the procedure lifecycle concept that appeared later. As such, it can be viewed as one that establishes an ATP. Yet, the approach pays scant attention to the transfer issue; just an example of the analytical procedure transfer by the SPhU criteria is provided. As is the case with the classical approach, that of the SPhU may be seen rather formal, overlooking the aspects that ensure reliable use of the procedure in the subsequent routine analysis. In parallel to the conventional policy, the SPhU approach does not focus on understanding the sources of variability and treat the phases of the procedure lifecycle separately.

Evidently, none of the approaches alone is entirely satisfactory.

Considering all the above, development of the strategy on the analytical procedure transfer that combines the benefits of known approaches to the issue seems sensible. To put the strategy to the test, a real object of the study is required.

Earlier, we developed and validated a UV-Vis spectrophotometric procedure for the desloratadine assay in film-coated tablets applying the SPhU and the lifecycle approach $[14,15]$. Therefore, it is of interest to use this procedure as an object of the present research.

In this work, we attempt to frame and pilot an optimised scientifically sound concept of the transfer of analytical procedures for assays by integrating the advantageous provisions of the conventional and lifecycle approaches to validation with the metrological base of the State Pharmacopoeia of Ukraine.

\section{Planning (methodology) of research}

The ultimate goal of the transfer is to test the capability of the analytical procedure to yield the analysis results that continuously satisfy the predefined ATP and verify its fitness for the routine use in the RU [9].

For the procedure transfer to be successful, a transfer design should ensure that all identified significant sources of variability are under control (have an acceptable amplitude), and any potential interferences do not significantly affect the measurement uncertainty in the RU. This is possible with the lifecycle principles such as analytical quality by design (AQbD), procedure lifecycle, quality risk management, knowledge management, ATP, analytical control strategy, and measurement uncertainty. In the lifecycle approach, the latter is suggested to serve as a scientific basis for the establishment of acceptance criteria and the assessment of results. The practical implementation of the uncertainty concept is explicitly described in the SPhU general texts Validation of Analytical Procedures (5.3.N.1) [10] and Statistical Analysis of Results of Chemical Experiment (5.3.N.2) (a linear model for the uncertainty budget, a principle of insignificance, etc.) [11].

Accordingly, first, it is rational to formulate a general strategy on the analytical procedure transfer based on the SPhU metrological approach and the advantageous lifecycle principles. Then, we need to design an experiment and establish acceptance criteria for the transfer of the analytical procedure for the desloratadine assay in film-coated tablets Alerdez based on the requirements for the target uncertainty of the procedure in the ATP (precision and accuracy), considering metrological parameters of the batch (the average content of desloratadine and its uncertainty). Finally, an assay of desloratadine should be conducted in the RU according to the developed experiment design, and the obtained values are to be assessed against the predetermined acceptance criteria.

In the event of failure to comply with any acceptance criterion, an investigation is required to be carried out to identify the root cause. If applicable, corrective actions should be taken depending on their nature.

An experiment needs to be designed considering the tasks prior accomplished in the $\mathrm{SU}$ at previous phases of the procedure lifecycle and the knowledge gained during the method development and validation. The transfer should deepen our understanding of the variability sources. If necessary, the control strategy should be refined.

\section{Materials and methods}

The research was performed on a pilot-scale batch of the pharmaceutical preparation Alerdez, film-coated 
tablets containing $5 \mathrm{mg}$ of desloratadine, manufactured by PJSC SIC “Borshchahivskiy CPP”, Ukraine, using a validated spectrophotometric procedure for desloratadine assay. The content of desloratadine per tablet is specified to be $\pm 5 \%$ of the labelled amount (from $4.75 \mathrm{mg}$ to $5.25 \mathrm{mg}$ ). The tablet mass is ca. $105 \mathrm{mg}$.

In the study, an analytical balance Mettler Toledo XP 205DR, Class A volumetric pipettes and flasks, PTFE filter with pre-filter $0.45 \mu \mathrm{m}$ cat. No. SYTG0602MNXX104 manufactured by MDI were used. Absorbance readings were measured on a UV-Vis spectrophotometer Lambda 25 equipped with a 1-cm cuvette (Perkin Elmer) at $282 \mathrm{~nm}$ and $350 \mathrm{~nm}$.

A desloratadine reference standard of the State Pharmacopoeia of Ukraine (SPhU Desloratadine RS) fitted for assays by UV-Vis spectrophotometry was used for the preparation of reference solution (assigned value: $99.7 \%$; target uncertainty: NMT $0.5 \%$, expressed as a one-sided $95 \%$ confidence interval).

All reagents met the requirements of the European Pharmacopoeia.

Test solution. Weigh and grind 20 tablets to a visually homogeneous fine powder using a mortar and pestle. Transfer an accurately weighed portion of the tablet powder equivalent to ca. $420 \mathrm{mg}$ to a $1000-\mathrm{mL}$ volumetric flask. Add $800 \mathrm{~mL}$ of $0.1 \mathrm{M}$ hydrochloric acid and sonicate for about $20 \mathrm{~min}$ with intermittent manual shaking. Cool and dilute the content of the flask to volume with the same solvent, and mix. Pass through a filter and use the filtrate.

Reference solution. Dissolve ca. $40 \mathrm{mg}$ of SPhU Desloratadine RS in $100 \mathrm{~mL}$ of $0.1 \mathrm{M}$ hydrochloric acid. Dilute $5.0 \mathrm{~mL}$ of the resulting solution with the same solvent to volume in a $100 \mathrm{~mL}$ volumetric flask.

Absorbance readings are performed alternately for each solution with the cuvette removal in at least three parallel replicates.

Calculate the content of desloratadine $\left(\mathrm{C}_{19} \mathrm{H}_{19} \mathrm{ClN}_{2}\right)$ per tablet $\left(X_{i}\right)$, in $\mathrm{mg}$, by the formula:

$$
X_{i}=\frac{A_{1} \times m_{0} \times P \times b \times 5 \times 1000}{A_{0} \times m_{1} \times 100 \times 100 \times 100}=\frac{A_{1} \times m_{0} \times P \times b}{A_{0} \times m_{1} \times 200},
$$

where $A_{l}-$ absorbance of the test solution, $\mathrm{AU}$; $A_{0}-$ absorbance of the reference solution, $\mathrm{AU} ; m_{0}-$ mass of the portion of SPhU Desloratadine RS, mg; $m_{l}$ - mass of the portion of the tablet powder, mg; $P$ - value assigned to SPhU Desloratadine RS, per cent; $b$ - average mass of tablet, $\mathrm{mg}$.

\section{Results \\ 4.1. Formulation of the concept of the optimal transfer of assay procedures}

As framed in the aim, we need to develop the alternative, scientifically sound principles of the transfer of analytical procedures for assays of active pharmaceutical ingredients (API) in finished drug products that should be consistent with the current ICH recommendations and take advantage of the lifecycle ap- proach and the developments of the State Pharmacopoeia of Ukraine.

Irrespective of whether the transfer of analytical procedures is viewed as a separate process (the classical approach) or an integral part of validation (the lifecycle approach), it rests upon validation results. Therefore, we approach the transfer in conjunction with the validation.

The ICH guideline on validation of analytical procedures lacks the scientifically justified acceptance criteria for validation that could powerfully demonstrate the suitability of the procedure for the intended use [3]. The approach does not expressly rely on the concept of uncertainty. For the transfer, the organisational steps are mainly presented. The criteria provided in the guidelines of the World Health Organization [2] are neither sciencebased nor recommended. They are not differentiated between the assays of substances and finished drug products, one- and two-sided assay limits, and various widths of two-sided specifications. This casts doubt on their reasonableness.

As the classical approach does not put emphasis on understanding the sources of variability, it may not bring them under effective control. On top of this, it addresses the events that take place over the procedure lifecycle (development, validation, transfer, etc.) as the separate processes that are not aimed at the subsequent routine use. This often leads to the formal, one-off verification of the fulfilment of the acceptance criteria. The possible outcome is that the procedure may not dependably perform in the RU [4].

Both the SPhU and lifecycle approaches to validation are aligned with the $\mathrm{ICH}$ guidelines but have distinct features that may complement the classical methodology and fill the gap. Further, we analyse some elements of the two to determine the provisions that should be incorporated into the optimal transfer concept.

The lifecycle approach to the transfer of analytical procedures has many advantages. It intends to adapt the ICH Q8 systematic guidance for the manufacturing process to the analytical procedure and employs the concept of measurement uncertainty. The approach focuses on the comprehension and control over the sources of variability. All phases of the procedure lifecycle, including the transfer, aim to ensure the correct use of the procedure in the subsequent routine analysis, which significantly increases its robustness. The special attention is paid to the study object specifics and its technology-related properties. These aspects may be overlooked when the classical or the SPhU approach is undertaken.

The SPhU approach relies on the pharmacopoeial decision rule that follows from the general notices and requirements of the United States Pharmacopeia (USP) [16], the European Pharmacopoeia (Ph. Eur.) [17], and the International Pharmacopoeia (IP) [18] stating that analytical variability is included in specifications. The Ph. Eur. and IP clarify that the conclusion on compliance is made without considering the measurement uncertainty, which is correct when the measurement uncertainty is sufficiently small compared to the width of the specification range [19]. With this in mind, the SPhU formulated a principle of insignifi- 
cance for the Test Uncertainty Ratio, which for a $95 \%$ confidence level, should be not more than 1:0.32. Under the SPhU approach, for assays in finished drug products with the specification range of $\pm 5 \%$, the target measurement uncertainty should be $5 \times 0.32=1.6 \%$. Thus, the only prerequisite for the $\mathrm{SPhU}$ approach is a $95 \%$ confidence level, which, however, is generally accepted. The SPhU approach makes it possible to decide with a $95 \%$ confidence level, whether the true analyte value lies within the specification range.

The stimuli article on the Analytical Target Profile [20] presents an example where requirements for the target measurement uncertainty are determined on the assumption that batches are manufactured to a nominal target value of $100 \%$ of label claim. It follows that a reliable decision on compliance may not be made in the event an analyte content is close to the extreme values of the specification limits. However, this is precisely the task that official quality control laboratories should handle. In our opinion, it is also of interest of manufacturers that operate in the GMP environment. Moreover, we are confident that pharmacopoeial procedures should be designed to cope with this task. From our perspective, the rationale for the target measurement uncertainty provided in the article is somewhat unconvincing.

The $\mathrm{Ph}$. Eur. and IP state that the limits are evaluated based on normal analytical practice [18, 21]. Similarly, the USP declares that acceptance criteria allow for analytical error [22]. A logical consequence of this is the necessity for all laboratories that use specifications established under the pharmacopoeial decision rule (which applies to all specifications of the USP, Ph. Eur., and IP) to comply with the normal analytical practice. This primarily concerns volumetric operations and standardisation of variation for analytical instruments. The EDQM issued guidelines [23] upon which the SPhU laid down requirements for the target measurement uncertainty for primary volumetric operations (the use of volumetric flasks and pipettes, transfer and graduated) [13]. Also, the SPhU defined requirements for RSD for the spectrophotometric methods for routine analyses proceeding from the interlaboratory experiment (not more than $0.52 \%$ ) and for the chromatographic methods for assays of finished drug products going from the requirements for the target measurement uncertainty, which allows monitoring over the implementation of normal analytical practice in the laboratory. The requirements have been used in proficiency testing schemes in Ukraine.

The approach of the SPhU enables predicting the actual maximum measurement uncertainty out from the text of the procedure, assuming that the specific sources of variability are negligible. Such a prognosis is obligatory in the preliminary design of the procedure. Besides, for the analysis result to be legitimate, the laboratory should ensure that the actual uncertainty for volumetric operations and analytical instruments agrees with the guidelines for normal analytical practice. Per the SPhU approach, the procedure is designed considering the requirements for normal analytical practice so that the predicted uncertainty does not exceed the target measurement uncertainty.

Having established requirements for normal analytical practice and monitoring their implementation in the laboratory may fundamentally change the goal of the precision study in the procedure lifecycle, which during validation turns to the confirmation that the variability in results meets the predefined criteria, i.e. there are no unanticipated and, therefore, uncontrolled interferences. The control over the observance with the normal analytical practice in the RU makes it possible to narrow down the transfer experiment size considerably.

The knowledge about the variability sources specific to the procedure should be gained during the development and validation of the procedure. In the transfer, it is sufficient to control that their amplitude has not critically increased in the RU. Note that the SPhU approach takes account of the fact that the amplitude of variability sources in the laboratory may vary greatly over time. From our experience, the precision of measurements largely depends on the technical condition of analytical instruments. Besides, random variability may be attributed to the work of the analyst due to both objective (e.g. expertise) and subjective (e.g. physical state) reasons. The SPhU approach ensures that the compliance of variability with the maximum allowable level regulated by normal analytical practice requirements is controlled throughout the procedure lifecycle.

In line with the classical policy, the lifecycle approach proposes to study precision in the RU in a shortterm experiment. From where we stand, this way seems biased since the results collected in a short-term experiment, such as transfer, do not reflect the maximum possible variation that may occur in the laboratory. Additionally, provided the normal analytical practice is monitored in the laboratory as per the SPhU, the control over the precision during the transfer duplicates the work already done in the RU. Satisfying recommendations of the SPhU provides an opportunity to unburden the RU significantly during the transfer by employing a confirmation approach (demonstration of the accordance of results with the predefined requirements in a minimal experiment) instead of the proof approach (studying precision in an extensive experiment). The observance with the normal analytical practice is controlled when the analytical procedure validation is carried out following the SPhU. It is verified in the development of the SPhU monographs. With the SPhU approach, the viewpoint of studying intra-laboratory precision may be rethought.

The revised draft general chapter <1220> [14] and the example of an ATP in work [20] suggest calculating intermediate precision "as the sum of the two components attributable to the different sources of variability: variation observed when an analytical procedure is used repeatedly to assess the same sample over a short period by a single analyst using the same equipment and variation that occurs when an analytical procedure is used in the same laboratory under random conditions such as different analysts, equipment, or days." We would like to point out that this approach has been recommended for 
biopharmaceutical applications in work [24]. As it is impossible to standardise a biological object, the consideration of the actual variation between experimental runs is required. The $\mathrm{SPhU}$ advances standardising the variation for all components of the analytical system, which is possible, and we believe necessary, in the case of chemical analyses.

The variation in the performance of various spectrophotometers is also under control in the SPhU approach. Our 18-year experience as the official proficiency testing provider in Ukraine makes us assert that for spectrophotometric measurements performed with the cuvette removal, the main source of deterioration in the precision results is often the dilapidated cuvette holder [25]. As far as we know, the measurements with the cuvette removal are the basic way of performing parallel measurements in quality control of medicines. Spectrophotometer manufacturers, however, control the precision of the absorbance measurement without removing the cuvette. In this regard, a national part to the monograph Absorption Spectrophotometry, Ultraviolet and Visible (2.2.25) that standardises RSD for 30 parallel measurements $(\leq 0.52 \%)$ has been introduced in the $\mathrm{SPhU}$ [26]. Obtained in the interlaboratory experiment and proved to be feasible for all modern spectrophotometers, this value ensures that requirements for the target measurement uncertainty of $1.6 \%$ are met when using the procedures in which a minimum of three parallel measurements and two sequential dilutions by taking aliquots are stipulated. Thus, if the SPhU approach is pursued, it becomes unnecessary to study the precision depending on the day, analyst, and spectrophotometer.

From the point of view of the authors, the lifecycle approach to partitioning variation between measurement runs remains incomplete in terms of the QbD concept since it may not lead to the understanding of sources of variability in interlaboratory precision, which is a prerequisite for their reliable control. In contrast, following the SPhU approach, one can understand and continuously control them. In view of the above, the intralaboratory precision calculation may be useful as an additional check on the conformity to normal analytical practice. However, carrying out such an extensive experiment during the transfer seems irrational.

The SPhU uses one-sided confidence intervals for a $95 \%$ confidence level to estimate uncertainty, which is generally accepted. The lifecycle approach advocates utilising tolerance intervals, including at the Procedure Performance Qualification Stage [20], which we consider non-transparent as it requires setting an additional parameter (proportion) as opposed to the confidence intervals. For tolerance intervals, both the confidence level and the proportion may be set somewhat arbitrarily. In the provided examples, the proportion of $60 \%$ and $90 \%$, and the level of confidence of $90 \%$ and $50 \%$ are used. This creates a risk of manipulating conclusions about the suitability of the procedure by adjusting parameters for tolerance intervals. Besides, the approach assumes that the experimental data from which the tolerance interval is calculated will belong to the same population as the future data. Given that the variation in the laboratory results may vary significantly over time, we view the use of tolerance intervals as an incorrect metrological model. Work [27] suggests evaluating precision from the data obtained in the stability study in the course of pharmaceutical development, i.e. acquired over a sufficiently long period, which ensures a valid estimate of precision. We find this approach rational.

Conventional approaches to the transfer of analytical procedures are classified into four types: comparative testing of the same batch by both the SU and the RU, co-validation between two or more laboratories, revalidation, and transfer waiver [28]. Although the first one is the most used technique for the transfer of procedures intended for assays, we consider comparative testing insufficiently effective without scientifically substantiated requirements for the target measurement uncertainty since it may not lead to the demonstration of the suitability of the procedure in the RU. Co-validation and revalidation in the RU are costly and time-consuming and, therefore, should be avoided whenever possible. The lifecycle approach overloads the RU with works that do not contribute to effective control over variability sources. From our point of view, the most effective technique is to establish metrological characteristics for the batch of the pharmaceutical preparation intended for transfer (the mean API value and the variation in API values between dosage units) in the SU and compare transfer results against them in the RU. In such instance, a batch of the pharmaceutical preparation acts as a reference standard, and small-size transfer results are compared with a large array of those obtained in the SU. Such an approach is unique as it is not considered in the classical and lifecycle systems.

Considering all the above, we define the concept of the optimal transfer of analytical procedures for assays as follows:

1. The transfer should be seen as an integral part of the overall procedure lifecycle that centres on ensuring a solid performance of the analytical procedure in the subsequent routine use and rests on the knowledge of variability sources acquired at the previous phases of the procedure lifecycle, bearing in mind that their amplitude may change over time. This should be considered in the analytical control strategy and the transfer experiment design. In routine use, the knowledge about variability sources should be refined, and the analytical control strategy should be adjusted if necessary.

2 . The maximum amount of work should be done in the SU prior to the transfer during an extensive longterm study of the procedure (knowledge-gathering, primarily, identification of significant sources of variability). The transfer should be aimed at confirming in a minimal experiment that the knowledge about the amplitude of the sources of variability is applicable to the RU. In the transfer process, the RU should demonstrate compliance with all acceptance criteria, which, with high reliability, indicates that the results obtained in the subsequent long-term use of the procedure will be accurate. The study of precision in the RU seems less practical as the results obtained in a short-term experiment are not representative. 
3. The laboratory (both the SU and the RU) should ensure observance with the normal analytical practice, e.g. as defined by the $\mathrm{SPhU}$.

4. The transfer should be conducted in the minimal experiment as follows*:

- verify whether the RU has sufficient knowledge to correctly reproduce the procedure (if necessary, a preliminary reproduction of the procedure in the RU should be done);

- check whether the analytical control strategy works in the RU;

- examine whether the requirements for the critical ATP criteria (precision and accuracy) are met.

*Note: applicable only if clause 3 is fulfilled.

The results can be used for the assessment of the variability sources that have not been identified in the SU.

5. The transfer should consider the specifics of the analysis object and the effect of the manufacturing process on those its properties that may lead to failure to comply with specifications. Evaluation of the variation stemmed from the technology and the study object is desirable for the assessment of transfer results. The comparison of the results obtained in the SU and the RU seems less effective.

6. Appropriate statistical tools and acceptance criteria should be used, e.g. those laid down in the $\mathrm{SPhU}$ (recommendations for the target uncertainty, accuracy, and normal analytical practice, the use of confidence intervals instead of tolerance intervals, and the use of normalised coordinates). The criteria should come from the quantitative estimation of the impact of the variability sources based on the risk of wrong conclusions on compliance.

\subsection{Experimental verification of the advocated concept of transfer}

Verification of the proposed methodology should be done on a real object of analysis according to the developed experiment design. All tests of the analytical control strategy must be performed. If necessary, the analytical procedure can be preliminarily reproduced in the RU. The transfer results should showcase the correctness of the analytical control strategy application, the compliance with the requirements for accuracy and measurement uncertainty, and the absence of new significant variability sources.

For the transfer, a validated analytical procedure for desloratadine assay in the pharmaceutical preparation Alerdez, $5 \mathrm{mg}$ desloratadine film-coated tablets, was chosen. The batch of desloratadine tablets that was extensively researched during the stability study was selected. The experiment was conducted considering the tasks completed at the previous phases of the analytical procedure.

Prior to the research, an ATP for the spectrophotometric procedure for the assay of desloratadine in the pharmaceutical preparation Alerdez was developed using the SPhU and the lifecycle approach. The procedure was optimised considering the uncertainty prognosis made from the SPhU requirements for normal analytical practice. Preliminary validation of the procedure was carried out on model solutions using the standardised scheme [14]. Technological variability was studied on the selected batch of desloratadine tablets to improve the averaging strategy and identify the risks of non-observance with the tests Assay and Uniformity of Dosage Units [29]. During pharmaceutical development, the risks stemmed from the specifics of the object of analysis, such as inhomogeneity of the test sample, were reviewed, and the precision of the procedure was studied.

All previous phases of the analytical procedure were based on the systematic approaches proposed in the lifecycle concept: procedure lifecycle, analytical target profile, quality risk management, control strategy, knowledge management, analytical quality by design, and measurement uncertainty. The SPhU metrological approach was employed for the organisation of the experiment and the establishment of acceptance criteria. Each step was used to enrich our knowledge about the sources of variability and develop a control strategy. The results were monitored for the consistency with the target uncertainty and the normal analytical practice.

In the present research, we need to balance the uncertainty of the API value for the batch of desloratadine tablets selected for the transfer considering the knowledge of the analytical and technological variability acquired at the previous phases of the procedure lifecycle, and to develop the acceptance criteria for the accuracy and precision of the analysis results taking into account the budget of the total variability. The SPhU and lifecycle approaches should be used to accomplish the tasks.

\section{Analytical control strategy}

An ATP for the procedure for assay of desloratadine in film-coated tablets was developed per the SPhU approach considering the risk of incorrect accept/reject decisions. The ATP is furnished in Table 1.

Table 1

The analytical target profile for the desloratadine assay procedure

\begin{tabular}{|l|c|}
\hline Specification limits, $\%$ & \pm 5 \\
\hline Risk of wrong (false negative) decisions $(\alpha), \%$ & 5 \\
\hline Target uncertainty $\left(U_{\text {Target }}\right), \%$ & 1.6 \\
\hline Bias, $\%$ & 0.51 \\
\hline
\end{tabular}

During the development and validation of the procedure, we identified the following significant variability sources that should be under control by the analytical control strategy: filtration efficacy, test sample inhomogeneity, and sample preparation uncertainty (associated with weighing and dilutions).

When using membrane filters with the same pore size $(0.45 \mu \mathrm{m})$ and type of material (PTFE), the difference in filtration efficacy was observed subject to the make of the filter. Moreover, the use of particular filters led to the slip of placebo components resulting in the opalescence of the solution and an unacceptable overestimation of the analysis results.

Since the opalescence effect resulted in the wavelength-independent absorption, it was proposed to measure the absorbance of the test solution at $350 \mathrm{~nm}$ as the 
analytical control strategy. The ratio of the absorbance of the test solution at $350 \mathrm{~nm}$ to the absorbance of the desloratadine reference solution at $282 \mathrm{~nm}$ (the maximum peak of the test solution), expressed in per cent, should be insignificant $(\leq 0.51 \%)$.

Due to the physical differences between the tablet coat and the tablet core, the underestimated assay results with the trend to an increase during the consecutive test portion taking were obtained. The first test portions were enriched by the film coat whereas the last ones - with the tablet core. The problem was solved by increasing the test portion mass used for the preparation of the test solution and implementing the analytical control strategy in the routine use of the analytical procedure (the control over the difference between the assay results obtained for the first and fourth test portions) [15].

The study of the analytical procedure precision from the stability data collected during the pharmaceutical development of the pharmaceutical preparation Alerdez showed that although the requirements for the target measurement uncertainty $\left(U_{\text {Target }}\right)$ were fulfilled, the variation in the absorbance for reference solutions $(1.1 \%)$ exceeded the criteria for normal analytical practice $(0.69 \%)$. The problem was solved by validating the specific absorbance $\left(A_{1}^{1}\right)$ for the reference solution that should be monitored as the analytical control strategy in the routine analysis (studied for six months on three spectrophotometers). The obtained confidence interval for the validated value of specific absorbance $(0.72 \%)$ was almost equivalent to the predicted value of uncertainty for normal analytical practice $(0.69 \%)$.

\section{bility}

\section{Budget of analytical and technological varia-}

To control the precision and accuracy of results during the transfer, the batch of the preparation Alerdez selected for the transfer of the analytical procedure was studied in depth during pharmaceutical development.

The following metrological parameters for the batch were established:

1. Grand mean value

$$
\bar{X}=98.9 \%
$$

The grand mean value was determined during the validation and stability studies.

2. Uncertainty of the API value in dosage units

The uncertainty of the API value in dosage units $\left(\Delta_{U D U}\right)$, expressed as a confidence interval for a single value, was calculated by the results of the test for Uniformity of Dosage Units performed by the Ph. Eur. (2.9.40) in nine repeats during pharmaceutical development.

$$
\begin{aligned}
& \Delta_{U D U}=R S D_{U D U} \times t(\alpha ; d f) \\
& \Delta_{U D U}=2.9 \times 1.66=4.82 \%
\end{aligned}
$$

where $R S D_{U D U}$ - estimated value of the technological variability; $t$-Student's $t$-test value; $\alpha$ - level of significance; $d f$ - number of degrees of freedom used to estimate the uncertainty of the API value in dosage units (here, $d f=89$ ).

Note that the technological variability was much greater than the analytical one. The measurement uncertainty calculated for nine model solutions in the validation study of linearity was $0.55 \%$, i.e. insignificant compared to $\Delta_{U D U}$ (by the $\mathrm{SPhU}$, the insignificance coefficient is 0.32 when comparing the confidence intervals). Therefore, the fact that the obtained estimate of the technological variability includes the actual analytical variability does not lead to the overestimation of the technological variability.

\section{Total variability in assay results}

The total variability in assay results $\left(\Delta_{\Sigma}\right)$ was predicted as follows:

$$
\begin{aligned}
& \Delta_{\Sigma}=\sqrt{U_{\text {Target }}^{2}+\left(\frac{\Delta_{U D U}}{\sqrt{n}}\right)^{2}} \\
& \Delta_{\Sigma}=\sqrt{1.6^{2}+\left(\frac{4.82}{\sqrt{20}}\right)^{2}}=1.94 \%
\end{aligned}
$$

where $\Delta_{U D U}$ - uncertainty of the API value in dosage units; $n$ - number of dosage units taken for averaging; $U_{\text {Target }}$ - target measurement uncertainty.

In Eq. (4), we used the target measurement uncertainty since the assay involves an operation of tablet grinding, and the uncertainty introduced by the sample heterogeneity cannot be predicted based on the requirements for normal analytical practice. In any case, the actual measurement uncertainty should not exceed the target measurement uncertainty, which is monitored by the analytical control strategy.

The total variability includes the uncertainty of the analytical procedure, the uncertainty of the API value in dosage units, and the corresponding averaging strategy (20 dosage units).

The budget of analytical and technological variability in the batch of desloratadine tablets used for the transfer is shown in Table 2.

Table 2

The budget of analytical and technological variability

\begin{tabular}{|l|c|}
\hline Type of variation & $\begin{array}{c}\text { Uncer- } \\
\text { tainty }\end{array}$ \\
\hline Analytical variability $\left(U_{\text {Target }}\right), \%$ & 1.6 \\
\hline $\begin{array}{l}\text { Technological variability }\left(\Delta_{U D U}\right), \% \\
\text { (for the individual tablet) }\end{array}$ & 4.82 \\
\hline $\begin{array}{l}\text { Total variability in assay results, }\left(\Delta_{\Sigma}\right), \% \\
\text { (for the mean of } 20 \text { tablets) }\end{array}$ & 1.94 \\
\hline Grand mean value $(\bar{X}), \%$ & 98.9 \\
\hline
\end{tabular}




\section{Design of the transfer experiment}

The transfer experiment design was developed, taking into consideration the ATP (requirements for uncertainty - precision and bias) and the developed analytical control strategy.
The verification of precision and bias in the RU was based on the budget of the analytical and technological variability made for the batch of Alerdez earmarked for the transfer (Table 2). The transfer experiment design is illustrated in Fig. 1.

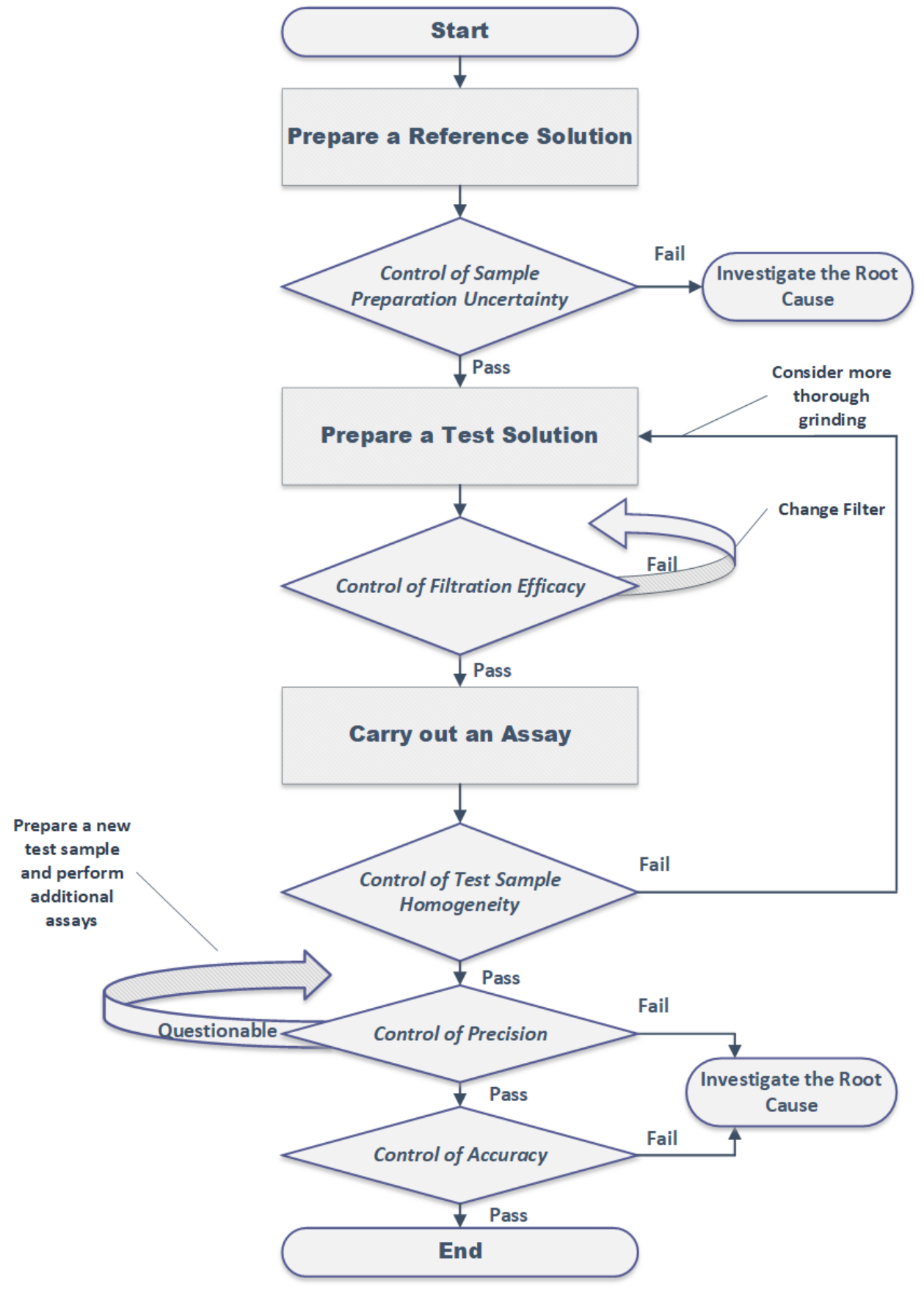

Fig. 1. Design of the transfer experiment

1. Control over the sample preparation uncertainty

We propose to control the sample preparation uncertainty by the specific absorbance of reference solutions, which allows us to monitor the compliance with the normal analytical practice.

Prepare a reference solution and measure the absorbance according to the analytical procedure. Calculate the specific absorption of the desloratadine reference solution ( $\left.A_{1}^{1}\right)$ by the formula:

$$
A_{1}^{1}=\frac{A_{282 n m} \times 1000 \times 1000}{c \times 100 \%},
$$

where $A_{282 n m}$ - absorbance of the desloratadine reference solution at $282 \mathrm{~nm}, \mathrm{AU} ; c$ - mass concentration of desloratadine in the reference solution, $\mu \mathrm{g} / \mathrm{mL}$.

Acceptance criteria: the obtained value of the specific absorption of the desloratadine reference solution should lie within the following limit: 


$$
A_{1}^{1} \in[3.202 \ldots 3.249] \times 10^{2}
$$

The limit was established for normal analytical practice. Nonconformity to the acceptance criteria may indicate an increased amplitude of variability from the controlled sources (analytical instruments, volumetric glassware, reference standards, uncertainty introduced by the analyst when performing standard procedures of sample preparation such as weighing, dilutions, etc.)

\section{Control over the membrane filtration efficacy}

Prepare a test solution and measure the absorbance at $350 \mathrm{~nm}$.

Acceptance criteria:

The absorbance of the test solution at $350 \mathrm{~nm}$ $\left(A_{350 n m}\right)$, AU, should be not more than the following limit:

$$
A_{350 m m} \leq 0.0032,
$$

where 0.0032 - coefficient that ensures that the background absorption is insignificant compared to the nominal absorbance for a $100 \%$ desloratadine solution.

The limit was established for the maximum value of bias by the ATP. Nonobservance with the acceptance criteria may be attributable to a slip of placebo components through the membrane filter, resulting in the systematic overestimation of the analysis results. If this a case, the filter batch or type should be changed.

\section{Control over the test sample homogeneity}

Prepare a test sample following the procedure. Make four test solutions from the test portions taken sequentially and perform assays.

Acceptance criteria:

The assay results obtained for the first and fourth test portions should not differ by more than $2.3 \%$ (Eq. 8) [15].

$$
\begin{aligned}
& \left|T S_{1}-T S_{4}\right| \leq \sqrt{2} \times U_{\text {Target }} ; \\
& \left|T S_{1}-T S_{4}\right| \leq 2.3 \%
\end{aligned}
$$

where $\mathrm{TS}_{1}$ - result of the assay in the first test solution, \%; $\mathrm{TS}_{4}-$ result of the assay in the fourth test solution, \%; $U_{\text {Target }}-$ target measurement uncertainty, \%.

Noncompliance with the acceptance criteria is highly likely the result of the insufficient test sample homogeneity. More thorough execution of tablet grinding is required. Increasing the time and the grinding force may reduce the inhomogeneity of the test sample.

\section{Control over the precision}

Analyse all test solutions prepared for Control over the test sample homogeneity. Calculate the confidence interval for a single value of the analysis results for four test solutions $\left(\Delta_{i}\right)$.

Acceptance criteria [15]:

If the confidence interval is lower or equal to $1.6 \%$, the precision is acceptable, and the RU demonstrates the compliance.
If the confidence interval is greater than $1.94 \%$, the precision is considered insufficient, and the RU is not compliant. The root cause should be investigated.

If the confidence interval is between $1.6 \%$ and $1.94 \%$, inclusive, the precision is considered questionable. If this is the case, a new test sample should be prepared using the next 20 dosage units, and additional assays should be performed. Calculation of the standard deviation for single assay results and the pooled confidence interval for all assay results should be made. If the pooled confidence interval is lower or equal to $1.6 \%$, the precision is considered acceptable and the performance of the RU compliant. Otherwise, the conclusion is made of insufficient precision.

In scenarios where the acceptance criteria are not met, the root cause should be investigated.

Failure to comply with the acceptance criteria may lie in the fact that there is a new source of variability in the RU or that known sources are out of control.

\section{Control over the accuracy}

For the test results obtained during Control over the precision, calculate the maximum difference between a single assay value and the grand mean.

Acceptance criteria:

The difference between the single assay value and the grand mean value should not exceed the total variability in assay results:

$$
\begin{aligned}
& \max \left|T S_{i}-\bar{X}\right| \leq \Delta_{\Sigma} \\
& \max \left|T S_{i}-\bar{X}\right| \leq 1.94 \%
\end{aligned},
$$

Noncompliance with the acceptance criteria may indicate a new source of variability in the RU or that known sources are out of control.

If both the precision and accuracy criteria are met, the transfer is considered successful.

\section{Results of the transfer}

The results of the transfer of the analytical procedure for the desloratadine assay in the pharmaceutical preparation Alerdez are furnished in Table 3.

The results presented in Table 3 indicate that the transfer of the analytical procedure for the assay of desloratadine in film-coated tablets Alerdez was a success.

The RU demonstrated an ability to perform the procedure as intended, which is evidenced by the compliance with the requirements for normal analytical practice and acceptance criteria. The membrane PTFE filter with pre-filter $0,45 \mu \mathrm{m}$ (MDI) was suitable for the purpose, and its replacement was not required. The sufficient homogeneity of the test sample was achieved following the observance with the transferred knowledge about tablet grinding. There was neither an increase in the amplitude variability nor a systematic shift in the assay results observed, i.e. no new variability sources were identified. 
Table 3

Results of the analytical procedure transfer

\begin{tabular}{|l|c|}
\hline \multicolumn{2}{|c|}{ Assay results } \\
\hline$X_{1}, \%$ & 98.3 \\
\hline$X_{2}, \%$ & 99.5 \\
\hline$X_{3}, \%$ & 99.7 \\
\hline$X_{4}, \%$ & 99.5 \\
\hline Mean, \% & 99.25 \\
\hline
\end{tabular}

Control over the sample preparation uncertainty

\begin{tabular}{|l|l|}
\hline Calculated specific absorption of the de- & 323.3
\end{tabular}

sloratadine reference solution $\left(A_{1}^{1}\right)$

\begin{tabular}{|l|c|}
\hline Deviation from the validated $A_{1}^{1}(322.6), \%$ & 0.22 \\
\hline Acceptance criteria, \% & $\leq 0.72$ \\
\hline Conclusion: & pass \\
\hline
\end{tabular}

Control over the membrane filtration efficacy

Ratio of the absorbance of the test solution at

0.16

$350 \mathrm{~nm}$ to the absorbance of the desloratadine

reference solution at $282 \mathrm{~nm}\left(A_{350 \mathrm{~nm}} / A_{282 \mathrm{~nm}}\right), \%$

\begin{tabular}{|c|c|}
\hline Acceptance criteria, $\%$ & $\leq 0.51$ \\
\hline Conclusion: & pass
\end{tabular}

Control over the test sample homogeneity

Difference between the assay values obtained 1.2

for the first and fourth test solutions

$\left(\left|T S_{1}-T S_{4}\right|\right), \%$

\begin{tabular}{l|c}
\hline Acceptance criteria, \% & $\leq 2.3$ \\
\hline Conclusion: & pass
\end{tabular}

Conclusion:

Confidence interval for a single value $\left(\Delta_{i}\right), \% \quad 1.51$

\begin{tabular}{l|c} 
Acceptance criteria, \% & $\leq 1.6$ \\
\hline Conclusion: & pass
\end{tabular}

\begin{tabular}{|c|c|}
\hline Conclusion: & pass \\
\hline \multicolumn{2}{|l|}{ Control over the accuracy } \\
\hline $\begin{array}{l}\text { Maximum deviation from the grand mean } \\
\text { value }\left(\max \left|T S_{i}-\bar{X}\right|\right), \%\end{array}$ & 0.8 \\
\hline Acceptance criteria, $\%$ & $\leq 1.94$ \\
\hline Conclusion: & pass \\
\hline
\end{tabular}

\section{Discussion}

The RU had sufficient experience in performing analytical procedures for assays by UV-Vis spectrophotometry, and preliminary reproduction of the procedure was not required. The experiment was conducted by the developed protocol design. The results obtained in the RU met all acceptance criteria.

The RU demonstrated that significant sources of variability identified in the SU were under control, and there was no critical impact of others on the measurement uncertainty. Consequently, there was no need to refine the analytical control strategy at the time of the transfer. However, it is vital to monitor the procedure performance in the routine use and refine the knowledge during the continued procedure verification stage.

It should be noted that the SPhU requirements for the target measurement uncertainty and accuracy that we propose to use for the transfer of analytical procedures are quite stringent. Their fulfilment in the laboratories adhering to the SPhU approach (virtually all laboratories of the pharmaceutical sector in Ukraine, both regulatory authorities and pharmaceutical companies) is ensured by personnel training and testing by inter- and intralaboratory proficiency testing programs specially developed by Ukrainian Scientific Pharmacopoeial Center for Quality of Medicines to control the observance with the normal analytical practice.

From the experience of the authors, there are commonly underestimated sources of variability that should be standardised and monitored such as the uncertainty introduced by the analyst, the inhomogeneity of the reference standard [30] and the test sample [15]. They are monitored by the developed analytical control strategy. Without their understanding, scientists may question the informativeness of assay results for narrow specification limits, primarily, for assays of drug substances $[31,32]$. Given that the laboratory is specially trained to observe the normal analytical practice, it can be decided that the requirement for the the target measurement uncertainty to be insignificant in relation to the narrowest specification limits of $\pm 5 \%$ of the API labelled amount specified for finished drug products is achievable.

We propose a novel way to confirm the correctness of the results obtained in the RU, where the mean API value in the tablet batch is established, and the budget of technological and analytical variability is balanced. First, it allows the analyst to control not only the precision but also the accuracy of the analysis results in the RU. Second, the knowledge of the variability budget makes the assessment of the result independent from the fact that the assay is carried out on the sample whose mean API value differs from the grand mean of the batch. An additional advantage of our approach is that the batch intended for the transfer may serve as a test item for personnel testing.

To our way of thinking, the deviation of the mean API value from the nominal one is primarily attributed to the average tablet mass deviation from the nominal value. This parameter is not standardised in official guidelines. As far as we are concerned, some pharmaceutical companies use an empirical criterion for deviation of $\leq 1.5 \%$. Therefore, the deviation of the API value from the nominal one can reach $1.5 \%$ under GMP conditions. From our perspective, it is crucial to consider the actual deviation of the grand mean from the nominal value when assessing the correctness of the results.

In the example provided in work [20], an assumption is made that an actual API value in the batch is $100 \%$. This contradicts our results obtained during the transfer of the analytical procedure for desloratadine assay in tablets. The actual deviation of the mean API value from the nominal content was about twice the SPhU criterion for the bias of $0.51 \%(\bar{X}=98.9 \%)$.

This allows us to conclude that the transfer concept and the acceptance criteria advocated in this paper are acceptable for the pharmaceutical preparation Alerdez. The concept may be applicable to the transfer of other analytical procedures for assays in finished drug products.

Study limitations. The success of the procedure transfer by the proposed strategy might be attributed to 
the fact that the pharmaceutical preparation Alerdez is a 'problem-free' research object. The technological and analytical variability exhibited by the studied object was relatively low, which guaranteed full agreement with the acceptance criteria. Yet, since the developed concept has not been tested on other pharmaceutical preparations, it is early to claim its universal applicability. There might be a need to make some adjustments.

Prospects for further research. Although feasible for the analytical procedures lacking or managing specific sources of variability, the SPhU requirements for bias are quite stringent. They may not be reached when special sample preparation techniques are required (e.g., extraction or chemical reaction with the analyte). The $\mathrm{SPhU}$ approach was not designed to relax the requirements for bias by tightening those for precision, not unlike the general monograph Statistical Tools for Procedure Validation $<1210>$ of the USP that suggests such a statistical tool for tolerance intervals [33]. In view of this, using a similar statistical tool for confidence intervals based on the SPhU approach is prospective.

\section{Conclusion}

The paper outlines the strengths and weaknesses of the conventional and lifecycle approaches to the method transfer and presents a new science-based concept of the successful transfer of analytical procedures for assays. The concept is consistent with the $\mathrm{ICH}$ recommendations, takes advantage of the lifecycle methodology (quality by design, quality risk management, procedure lifecycle, control strategy, and knowledge management), and employs the metrological developments of the State Pharmacopoeia of Ukraine to the experiment design and acceptance criteria (requirements for target measurement uncertainty, accuracy and precision of the analytical procedure, observance with normal analytical practice in the laboratory, and the use of onesided $95 \%$ confidence intervals).
The devised strategy excludes proving precision during the transfer (in the short-term experiment). We believe that it is more rational to assess the precision from the stability study (in the long-term experiment) and refine the knowledge in the routine use. Provided the normal analytical practice (the maximum permissible variability attributed to analytical instruments and the work of analysts) is observed, the transfer experiment design may be narrowed down to the confirmation in the minimal experiment that the amplitude of the significant variability sources identified during the development and validation of the analytical procedure lies within the predefined range.

We have designed and successfully completed the transfer of the validated analytical procedure for desloratadine assay in film-coated tablets following the embraced concept. For the batch intended for the transfer, the budget of analytical and technological variability was balanced. The deviation of a single assay result from the grand mean was proposed to use as the criterion for accuracy during the transfer. As the criterion for precision, the requirement for a one-sided confidence interval for a series of assay results not to exceed the target uncertainty of the procedure was used.

The findings of the research support the proposed strategy. All requirements established in the analytical control strategy for significant variability sources (filtration efficacy, test sample homogeneity, and sample preparation uncertainty) and the analytical target profile for precision and accuracy were fulfilled in the receiving unit. However, given that the present study has been carried out on a 'problem-free' pharmaceutical preparation, further investigation is required.

\section{Conflict of interests}

The authors declare that they have no conflict of interests.

\section{References}

1. Technology Transfer: ISPE Good Practice Guide 3rd Edition (2018). Tampa: International Society for Pharmaceutical Engineering.

2. Technical Report Series, No. 961 (2011). Transfer of technology in pharmaceutical manufacturing. Geneva: World Health Organization.

3. Martin, G., Barnettm K., Burgess, C. et. al. (2013). Lifecycle Management of Analytical Procedures: Method Development, Procedure Performance Qualification, and Procedure Performance Verification. Pharmacopeial Forum, 39 (5). Available at: http://www.usppf.com/pf/pub/index.html

4. Ermer, J., Limberger, M., Lis, K., Wätzig, H. (2013). The transfer of analytical procedures. Journal of Pharmaceutical and Biomedical Analysis, 85, 262-276. doi: http://doi.org/10.1016/j.jpba.2013.07.009

5. Kaminski, L., Schepers, U., Wätzig, H. (2010). Analytical method transfer using equivalence tests with reasonable acceptance criteria and appropriate effort: Extension of the ISPE concept. Journal of Pharmaceutical and Biomedical Analysis, 53 (5), 1124-1129. doi: http://doi.org/10.1016/j.jpba.2010.04.034

6. Validation of Analytical Procedures: Text And Methodology Q2R1 Current Step 4 version: ICH Harmonised Tripartite Guideline

(2005). The International Conference on Harmonisation of Technical Requirements for Registration of Pharmaceuticals for Human Use.

7. Nethercote, P., Borman, P., Bennett, T., Martin, G. (2010). QbD for Better Method Validation and Transfer. Pharma Manufacturing. Available at: www.pharmamanufacturing.com/articles/2010/060.html

8. Martin, G., Barnettm K., Burgess, C. et. al (2017). Proposed New USP General Chapter: The Analytical Procedure Lifecycle <1220>. Pharmacopeial Forum, 43 (1). Available at: https://www.uspnf.com/notices/1220-analytical-procedure-lifecycle

9. United States Pharmacopeia (2020). <1220> Analytical Procedure Lifecycle. Pharmacopeial Forum, 46 (5).

10. Derzhavna Farmakopeia Ukrainy. Vol. 1 (2015). 5.3.N.2. Validatsiia analitychnykh metodyk i vyprobuvan. Kharkiv: Derzhavne pidpryiemstvo «Ukrainskyi naukovyi farmakopeinyi tsentr yakosti likarskykh zasobiv», 910-929.

11. Derzhavna Farmakopeia Ukrainy. Dopovnennia 2 (2018). 5.3.N.1. Statystychnyi analiz rezultativ khimichnoho eksperymentu. Kharkiv: Derzhavne pidpryiemstvo «Ukrainskyi naukovyi farmakopeinyi tsentr yakosti likarskykh zasobiv», 77-112.

12. Leontiev, D., Volovyk, N. (2016). Specificity of application of the uncertainty concept to the decision on compliance of medicines. Arhiv za farmaciju, 66, 207-208. 
13. Derzhavna Pharmakopeya Ukrayini. Vol 1 (2015). Kharkiv: DP «Ukrayinskiy naukoviy farmakopeyniy tsentr yakosti likarskih zasobiv», 1128

14. Leontiev, D., Petrus, V., Volovyk, N., Gryzodub, O. (2020). Validation of the Spectrophotometric Procedure For Desloratadine Assay in Tablets Applying The Uncertainty Concept of the State Pharmacopoeia of Ukraine. EUREKA: Health Sciences, 6, 74-87. doi: http://doi.org/10.21303/2504-5679.2020.001527

15. Leontiev, D., Petrus, V., Volovyk, N., Gryzodub, O. (2020). A study of the influence of the test sample inhomogeneity on variability in assay results of desloratadine in film-coated tablets. ScienceRise: Pharmaceutical Science, 5 (27), 43-51. doi: http://doi.org/10.15587/2519-4852.2020.215287

16. The United States Pharmacopeia (2020). USP General Notices and Requirements: 4.10.20. Acceptance Criteria. Available at: https://www.uspnf.com/sites/default/files/usp_pdf/EN/USPNF/usp-nf-notices/usp38_nf33_gn.pdf

17. European Pharmacopoeia (2019). General Notices. 1.4. Strasbourg: European Directorate for the Quality of Medicines, 1-11.

18. World Health Organization (2008). The international pharmacopoeia. Geneva: World Health Organization, Dept. of Essential Medicines and Pharmaceutical Policies.

19. EURACHEM/CITAC (2015). Setting and Using Target Uncertainty in Chemical Measurement. Available at: http://www.citac.cc/STMU_2015_EN.pdf

20. Barnet, K., McGregor, P. et. al. (2017). Analytical Target Profile: Structure and Application Throughout the Analytical Lifecycle. Pharmacopeial Forum, 42 (5).

21. European Pharmacopoeia (2019). Strasbourg: European Directorate for the Quality of Medicines. Available at: https://www.edqm.eu/en/european-pharmacopoeia-ph-eur-10th-edition

22. The United States pharmacopeia 43. The National formulary 38 (2019). Rockville: United States Pharmacopeial Convention, Inc.

23. European Directorate for the Quality of Medicines \& HealthCare (2015). Strasbourg: EDQM. Available at: https://www.edqm.eu/sites/default/files/technical_guide_for_the_elaboration_of_monographs_7th_edition_2015.pdf

24. Nijhuis, M. B., Van den Heuvel, E. R. (2007). Closed-Form Confidence Intervals on Measures of Precision for An Interlaboratory Study. Journal of Biopharmaceutical Statistics, 17 (1), 123-142. doi: http://doi.org/10.1080/10543400601001519

25. Grizodub, A., Zvolinskaya, N., Leontev, D. et. al. (2004). Vosproizvodimost farmakopeynyih spektrofotometricheskih metodik kolichestvennogo opredeleniya lekarstvennyih sredstv v raznyih laboratoriyah. Farmakom, 2, 20-34.

26. Derzhavna Farmakopeia Ukrainy. Vol. 1 (2015). 2.2.25N Absorbtsiina spektrofotometriia v ultrafioletovii ta vydymii oblastiakh. Kharkiv: Derzhavne pidpryiemstvo «Ukrainskyi naukovyi farmakopeinyi tsentr yakosti likarskykh zasobiv», 78-81.

27. Ermer, J., Arth, C., De Raeve, P., Dill, D., Friedel, H.-D., Höwer-Fritzen, H. et. al. (2005). Precision from drug stability studies. Journal of Pharmaceutical and Biomedical Analysis, 38 (4), 653-663. doi: http://doi.org/10.1016/j.jpba.2005.02.009

28. United States Pharmacopeia 40 (2017). <1224> Transfer of Analytical Procedures. Rockville: The United States Pharmacopeial Convention, 1778-1780.

29. Petrus, V., Leontiev, D., Volovyk, N., Grizodub, O. (2019). A technological variation forecast in the industrial production of desloratadine tablets. Farmakom, 1 (2), 37-48.

30. Volovyk, N., Leontiev, D., Gryzodub, O. (2019). The approach of the State Pharmacopoeia of Ukraine to the homogeneity study of pharmacopoeial reference standards. 13th International symposium on pharmaceutical reference standards, U.S. Pharmacopeial Convention (USP) \&EDQM, Council of Europe. Strasbourg. Available at: https://www.edqm.eu/sites/default/files/posters-referencesubstances-symposium-irss-march2019.pdf

31. Görög, S. (2005). The sacred cow: the questionable role of assay methods in characterising the quality of bulk pharmaceuticals. Journal of Pharmaceutical and Biomedical Analysis, 36 (5), 931-937. doi: http://doi.org/10.1016/j.jpba.2004.06.025

32. Elder, D. (2014). Are HPLC-UV Methods Fit for Purpose as True Arbiters of Quality for APIs? Available at: https://www.americanpharmaceuticalreview.com/Featured-Articles/163576-Are-HPLC-UV-Methods-Fit-for-Purpose-as-True-Arbiters-ofQuality-for-APIs/

33. The United States Pharmacopeia (2020). <1210> Statistical tools for procedure validation.

Received date 05.11.2020

Accepted date 08.12.2020

Published date 30.12.2020

Natalia Volovyk, PhD, Department of Validation and Reference Standards, State Enterprise "Ukrainian Scientific Pharmacopoeia Center for the Quality of Medicines”, Astromonichna str., 33, Kharkiv, Ukraine

Dmytro Leontiev, Doctor of Pharmaceutical Sciences, Senior Researcher, Head of Department, Department of Validation and Reference Standards, State Enterprise "Ukrainian Scientific Pharmacopoeia Center for the Quality of Medicines", Astromonichna str., 33, Kharkiv, Ukraine, 61085, Professor, Department of Pharmaceutical Chemistry, National University of Pharmacy, Pushkinska str., 53, Kharkiv, Ukraine, 61002

E-mail: leontievd@yahoo.com

Vasyl Petrus, Postgraduate Student, Department of Pharmaceutical Chemistry, National University of Pharmacy, 53, Pushkinska Str., Kharkiv, Ukraine, 61002, Engineer, Research and Implementation Laboratory, PJSC SIC «Borshcha hivskiy CPP», Myru str., 17, Kyiv, Ukraine, 03134, E-mail: petrus.vasyl@gmail.com

Oleksandr Gryzodub, Doctor of Chemical Sciences, Professor, Chief Researcher, Department of State Pharmacopoeia of Ukraine, State Enterprise "Ukrainian Scientific Pharmacopoeia Center for the Quality of Medicines”, Astromonichna str., 33, Kharkiv, Ukraine, 61085, E-mail: o.gryzodub@gmail.com

Yurii Pidpruzhnykov, Doctor of Pharmaceutical Sciences, Professor, Chief Researcher, LLC "Chemical company" Spoluka ", Murmanska str., 5, Kyiv, Ukraine, 02660, E-mail: gmpinsp@ gmail.com 